\title{
Safe Trajectories for Autonomous Rendezvous of Spacecraft
}

\author{
Louis Breger* and Jonathan P. How ${ }^{\dagger}$ \\ MIT Department of Aeronautics and Astronautics
}

\begin{abstract}
Autonomous spacecraft rendezvous is an enabling technology for many future space missions, but anomalies in recent flight experiments suggest that safety considerations will play a critical role in mission success. This paper presents a method for online generation of safe, fuel-optimized rendezvous trajectories that guarantee passive collision avoidance for a large class of anomalous system behaviors. We examine the cost of imposing safety as a problem constraint and of additional constraints that guarantee infinite horizon passive collision avoidance while enabling future docking retries. A convex collision avoidance formulation was introduced and determined to provide computationally fast solutions at a small fuel expense. Several examples using both rotating and non-rotating targets are presented to demonstrate the overall benefits of incorporating these safety constraints when compared to nominal trajectory design techniques.
\end{abstract}

\section{Introduction}

Autonomous spacecraft rendezvous is an enabling technology for many future space missions. ${ }^{1}$ Autonomous rendezvous has been used for docking with Mir, ${ }^{2}$ and more recently on the ETS-VII ${ }^{3}$ and $\mathrm{DART}^{4,7}$ missions. However, anomalies occurred during both of these last two missions. In the case of ETS-VII, multiple anomalies caused safe mode entries over the course of the mission, at least one of which caused a preprogrammed maneuver to move the spacecraft $2.5 \mathrm{~km}$ from its target. The anomaly in the DART mission is thought to have resulted in excess fuel expenditures and appears to have caused an on-orbit collision. ${ }^{7,5,6}$ Thus recent experience suggests that presently, autonomous rendezvous and docking is neither a mature, nor a safe, technology. Designing approach trajectories that guarantee collision avoidance for some common failures could simultaneously decrease the likelihood of catastrophic failures in which both spacecraft are destroyed and increase the likelihood that future attempts at docking succeed. This paper introduces a method for generating fueloptimized rendezvous trajectories online that are safe with respect to a large class of possible spacecraft anomalies.

Numerous methods of generating rendezvous trajectories exist in the literature and encompass a wide range of rendezvous scenarios. ${ }^{8,9,10,24,11}$ These papers consider rendezvous from many perspectives, often taking into account complicated collision avoidance constraints, nonlinear rotational dynamics, and fuel efficiency. Another perspective to be considered when designing trajectories is

${ }^{*}$ Research Assistant, MIT Department of Aeronautics and Astronautics, 1breger@mit.edu

${ }^{\dagger}$ Associate Professor, MIT Department of Aeronautics and Astronautics, jhow@mit.edu 
safe behavior. ${ }^{11,12,13,14}$ Safety in the context of spacecraft rendezvous and docking is typically with respect to collision avoidance following some type of failure. The approach in Ref. 14 creates trajectories which naturally tend to drift away from the target spacecraft in the absence of thrusting. This method can guarantee safety for thruster failures, but is not fuel-optimized and does not apply to more complicated docking situations in which those trajectories cannot be used for nominal rendezvous. Alternately, Refs. 11 and 12 develop the safety circle method, in which a nearby orbit with a relative invariant trajectory is established that allows safe long-term observation before docking, however this approach is not fuel optimized and does not propose a specific docking path. A method proposed in Ref. 13 optimizes both safety and fuel using genetic algorithms. This approach treats safety as a goal rather than a constraint and thus, cannot assure that the resulting trajectory would be safe. Ref. 24 plans safe trajectories using potential functions, but the approach is computationally intensive and limited to static obstacles. Various types of safety have been considered in the design of UAV trajectories, but these focused on creating trajectories that are safe under nominal operating conditions (e.g., safety from adversaries, uncertain terrain). ${ }^{15,16}$

This paper defines a safe trajectory as an approach path that guarantees passive collision avoidance in the presence of a class of anomalous system behaviors. Note that this definition of safety is more restrictive than guaranteeing nominal collision avoidance. Instead, it guarantees that for a range of faults, no collisions will occur even if the chaser spacecraft cannot use thrusters, computers, or communications equipment. The rationale behind choosing this passive abort strategy is threefold: (a) passive abort can protect against a large set of possible system failures simultaneously; (b) an abort trajectory that does not require fuel use guarantees that remaining fuel will not be expended rapidly to increase spacecraft separation distance, thereby increasing the likelihood that future docking attempts can occur; and (c) passive abort guarantees thrusting will not be used in close proximity to the target during an anomaly, thereby eliminating the danger of plume impingement during an automatic safe-mode maneuver.

The following sections review a method for generating fuel-optimized trajectories from linearized relative dynamics and develop a novel approach for guaranteeing those trajectories will be safe. Several examples of safe trajectories generated for docking with both rotating and nonrotating target spacecraft establish that adding safety constraints does not result in significantly increased in fuel use. Next, we examine additional constraints to guarantee desirable infinite horizon passive collision avoidance and ease of future docking attempts. To address online-implementation considerations, a convex formulation of the safety problem is introduced that trades some performance for large computation reductions.

\section{Online Trajectory Optimization for Autonomous Rendezvous and Docking}

A trajectory generated through online optimization can be designed by choosing the system inputs that produce that trajectory. For a linear system, methods for incorporating and propagating the effects of inputs are well-known. The trajectory optimization formulation in this chapter is presented in the context of linear time-invariant dynamics, but there is no inherent restriction in the formulation preventing the use of time-varying dynamics. ${ }^{22}$ Typically, in a rendezvous 
situation, spacecraft would be in sufficiently close proximity to use Hills equations, ${ }^{21}$ but GVEbased approaches ${ }^{18}$ can be used for more widely separated situations. Given a chaser satellite whose state is $\mathbf{x}_{k}$ at time $k$ where the state $\mathbf{x}$ is defined as

$$
\mathbf{x}=\left[\begin{array}{llllll}
x & y & z & v_{x} & v_{y} & v_{z}
\end{array}\right]^{T}
$$

where $x, y, z, v_{x}, v_{y}$, and $v_{z}$ are the positions and velocities of a chaser satellite in the radial, in-track, and cross-track axes of an LVLH frame, respectively, the dynamics of the system are

$$
\mathbf{x}_{k+1}=A_{d} \mathbf{x}_{k}+B_{d} \mathbf{u}_{k}
$$

where $A_{d}$ is the state transition matrix for a single time step, $B_{d}$ is the discrete input matrix for a single time step, and $u_{k}$ is the input vector at step $k$ which is defined as

$$
\mathbf{u}=\left[\begin{array}{lll}
u_{x} & u_{y} & u_{z}
\end{array}\right]^{T}
$$

where $u_{x}, u_{y}$, and $u_{z}$ are the inputs in the axes indicated by the subscripts in the LVLH frame. Given an initial state $x_{0}$, the state at any future step $k$ is ${ }^{19}$

$$
\mathbf{x}_{k}=A_{d}^{k} \mathbf{x}_{0}+\left[\begin{array}{lllll}
A_{d}^{k-1} B_{d} & A_{d}^{k-2} B_{d} & \ldots & A_{d} B_{d} & B_{d}
\end{array}\right]\left[\begin{array}{c}
\mathbf{u}_{0} \\
\vdots \\
\mathbf{u}_{k-1}
\end{array}\right]
$$

or in condensed notation

$$
\mathbf{x}_{k}=A_{d}^{k} \mathbf{x}_{0}+\Gamma_{k}\left[\begin{array}{c}
\mathbf{u}_{0} \\
\vdots \\
\mathbf{u}_{k-1}
\end{array}\right]
$$

where $\Gamma_{k}$ is the discrete convolution matrix in Eq. 4. Since the states are expressed in terms of the inputs, an optimization can be formed that both optimizes and constrains the inputs and constrains the states of the system. The cost function for this optimization over $N$ steps is

$$
J=\min _{\mathbf{u}_{0}, \ldots, \mathbf{u}_{N-1}} \sum_{i=0}^{N-1}\left\|\mathbf{u}_{i}\right\|_{1}
$$

where the 1-norm cost is used to capture the expenditure of $\Delta V$ from axial thrusters. At each step $k$, it is possible to constrain the state at that time to lie inside a convex region

$$
A_{k} \mathbf{x}_{k} \leq b_{k}
$$

where $A_{k}$ is an $m \times 6$ matrix and $b_{k}$ is a vector that together capture a set of linear constraints on the state. Alternately, the state $\mathbf{x}_{k}$ could be constrained to lie outside a region through the use of 
binary variables ${ }^{8}$

$$
\begin{aligned}
A_{k} \mathbf{x}_{k} & \leq b_{k}+M \mathbf{y}_{k} \\
\left\|\mathbf{y}_{k}\right\|_{1} & \leq m-1
\end{aligned}
$$

where $\mathbf{y}_{k}$ is a vector whose elements are constrained to be 0 or 1 , and $M$ is a large number on the scale of values taken by elements of $\mathbf{x}$. This "Big M" method of collision avoidance works by allowing, at most, all but one of the collision avoidance constraints to be relaxed. A constraint is relaxed when the binary variable associated with it is set to 1 , thereby making the right-hand side of the inequality very large and guaranteeing constraint satisfaction. Since at least one constraint is always guaranteed to not be relaxed, collision avoidance is assured (e.g., knowing that one is outside of one side of a box is sufficient information to guarantee that one is not in the box).

The inputs at each time step can also be directly constrained using

$$
\mathbf{u}_{\min _{k}} \leq \mathbf{u}_{k} \leq \mathbf{u}_{\max _{k}}
$$

where $\mathbf{u}_{\min _{k}}$ and $\mathbf{u}_{\max _{k}}$ are vector bounds on the values of $\mathbf{u}_{k}$. Typically, the minimum thrust at all times would be $-\mathbf{u}_{\max _{k}}$. A detailed description of the full matrix forms used in linear trajectory optimizations for space vehicles can be found in Refs. 22 and 8.

\section{Safety Formulation}

The trajectories generated by the constraints in Section II will observe constraints and use minimal fuel to arrive at a rendezvous location. However, as is typical of optimal paths, the trajectories will approach constraint boundaries and generally be sensitive to uncertain behavior. Refs. 17 and 23 describe computationally feasible methods of generating trajectories online that are robust to process and sensing noise. This section presents an approach for generating trajectories that are safe with respect to a class of system failures. While it would be desirable to avoid collisions and successfully complete docking in the presence of any system failure, it is unlikely that such a scenario is possible. Instead, a large subset of all possible failures is used, including guidance system shutdowns, which encompasses thruster failures, computer anomalies, and loss of sensing. The response to these types of failures would be a guidance system shut down in which the chaser vehicle would go into a safe mode with all its thrusters turned off. Safety to this class of failures is called passive abort safety, because any rendezvous can be aborted using no thrusting. Passive abort safety guarantees collision avoidance for any failure that can be identified and responded to by disabling thrusters before the spacecraft trajectory is affected. This type of safety does not include failures in which a thruster fails on.

A consequence of passive abort is that if thrusters are disabled at any step $T$, counted from the start of the plan, during the trajectory implementation, then the thrusters will remain failed until the last step $N$ of the plan. Clearly choosing $N-T$ to be small (i.e., only constraining steps toward 
the end of the horizon to be safe ${ }^{\mathrm{a}}$ ) puts fewer constraints on the trajectory optimization than a large value, but it assumes that more of the plan will be successfully implemented. Conversely, a large $N-T$ is a more conservative approach to safety and more tightly constrains the optimization. The choice of which steps in the plan that are constrained to be safe depends on the specific characteristics of the spacecraft and the mission requirements. The objective of this paper is to present a systematic way of embedding the safety goals into the path planning problem so that the designer can evaluate the trade-offs associated with choosing $T$.

Note that enabling passive safety abort at the end of a trajectory can eliminate potential plume impingement conflicts that may result from last-minute safe-mode maneuvers. A further benefit of guaranteeing safety at the end of the trajectory is that it gives controllers an immediate safe exit from the docking procedure during the period when the spacecraft are in the closest proximity and there is the least time available to plan emergency maneuvers.

The discrete convolution approach used in Eq. 4 can be used to predict the state of the chaser at step $k$ in the planning horizon in the event of a failure at time $T$, by considering all inputs after and including the input at time $T$ to be zero

$\mathbf{x}_{F T_{k}}=A_{d}^{k} x_{0}+\left[\begin{array}{llllllll}A_{d}^{k-1} B_{d} & A_{d}^{k-2} B_{d} & \ldots & A_{d}^{T-2} B_{d} & 0 \times A_{d}^{T-1} B_{d} & \ldots & 0 \times A_{d} B_{d} & 0 \times B_{d}\end{array}\right]\left[\begin{array}{c}\mathbf{u}_{0} \\ \vdots \\ \mathbf{u}_{k-1}\end{array}\right]$

where $\mathbf{x}_{F T_{k}}$ is the state of the chaser spacecraft at some step $k$ in the planning horizon after a failure occurred at step $T$. The value of a failure state after the planning horizon is found through open-loop propagation of the state at time $N$

$$
\mathbf{x}_{F T_{k}}=A_{d}^{k-N} \mathbf{x}_{F T_{N}} \quad \text { for } k \geq N
$$

Passive collision avoidance is achieved by adding constraints on the failure states of the spacecraft. Define the set of position states occupied by the target as $\mathcal{T}_{k}$, which can describe any polytopic region of position states, convex or otherwise. Safety can be guaranteed by introducing the set of constraints

$$
\mathbf{x}_{F T_{k}} \notin \mathcal{T}_{k} \quad \forall k \in\{T+1 \ldots N+S\}
$$

where $S$, the safety horizon, is some number of time steps after the end of the planning horizon that trajectories resulting from failures must remain safe. The constraints in Eq. 13 are then imposed for $T \in \mathcal{F}$ where $\mathcal{F}$ is the set of every potential failure time at which the system must guarantee collision avoidance for guidance shutdowns. The parameters to be chosen in this safety formulation are $\mathcal{F}$ and $S$. This choice of parameters is highly dependent on the requirements of a particular space mission. The advantage of choosing to be safe for a large number of steps and for a long safety horizon is improved likelihood of preventing a catastrophic failure scenario in which the chaser and target collide. However, imposing many safety constraints greatly reduces the number of potential

\footnotetext{
${ }^{a}$ All subsequent mentions of safety are with respect to passive abort safety.
} 
solution trajectories and as a result, likely reduces fuel efficiency. The tradeoff between safety and fuel efficiency is discussed in the scenarios in the next section.

\section{Scenarios}

The rendezvous and docking scenario to be examined in this paper involves a target spacecraft being docked with and a chaser spacecraft maneuvering to achieve that docking. Figure 1 shows a target spacecraft that lies at the center of an local frame. A line of sight (LOS) cone protrudes from the target spacecraft and it is required that rendezvous remain within this line of sight cone for vision based sensing. At the interface between the LOS cone and the target is a docking port (rectangular platform). In the rotating case (Figure 2) the axis of rotation is the long axis of the spacecraft and the rotation rate is orbital. The choice of rotation axis and rate was arbitrary and only enter the optimization through their effect on the time-varying constraints imposed for LOS requirements, docking, and safety. The LOS requirements are

$$
A_{\mathrm{LOS}_{k}} \mathbf{x}_{k} \leq b_{\mathrm{LOS}_{k}} \forall k=1 \ldots N
$$

where $A_{\mathrm{LOS}_{k}}$ and $b_{\mathrm{LOS}_{k}}$ describe the states within the LOS cone at a step $k$ in the planning horizon. The terminal constraint is

$$
A_{\operatorname{Term}_{N}} \mathbf{x}_{N} \leq b_{\operatorname{Term}_{N}}
$$

where $A_{\text {Term }_{k}}$ and $b_{\text {Term }_{k}}$ describe the states the spacecraft must occupy at the end of the planning horizon to achieve safe docking. These constraints can be both on position (e.g., enter a region within reach of a grappling arm) or and on velocity (e.g., dock within a velocity range that produces acceptable stress on the docking port). In addition, time-varying bounds are introduced on the maximum thrusting levels in order to ensure large thrusts are not planned for the period immediately before docking. The safety constraints in Eq. 13 are imposed for a quarter of the planning horizon. In the examples, an orbit with frequency $n=0.001 \mathrm{rad} / \mathrm{s}$ is used and is discretized into 20 steps and the set of inputs that can fail is $T \in\{14 \ldots 19\}$. The planning horizon is full orbit. The chaser spacecraft has a mass of $45 \mathrm{~kg}$ and a maximum acceleration of $10^{-3} \mathrm{~m} / \mathrm{s}^{2}$ during the first 17 steps of the plan and $10^{-5} \mathrm{~m} / \mathrm{s}^{2}$ for the last 3 steps. In these examples, the safety horizon is a full orbit.

\section{A. Case 1: Stationary Target Satellite}

An optimized trajectory with no safety constraints for the stationary target case is shown in Figure 3. The initial trajectory to the docking port roughly corresponds to a two impulse V-bar (in-track) ${ }^{11}$ approach. The nominal trajectory is marked with $\bullet$ and the failure trajectories with $\times$. Failure trajectories, those trajectories which would occur in the event of a guidance shutdown, corresponding to the last five possible inputs are shown. Several of the failure trajectories overlap, a condition which corresponds to the nominal input at a step already being zero thrust. All of the failure trajectories clearly impact the target spacecraft. Figure 4 shows the same rendezvous 


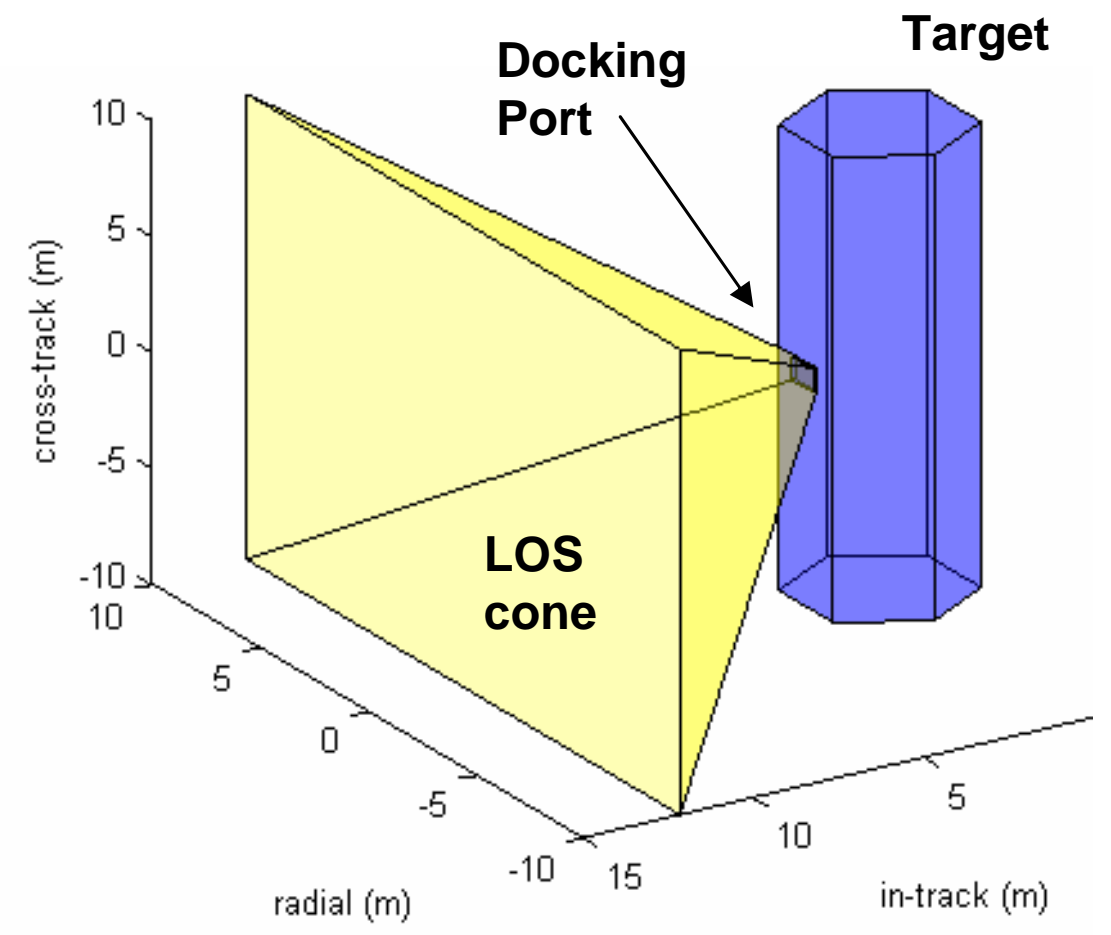

Fig. 1: Target spacecraft and docking configuration

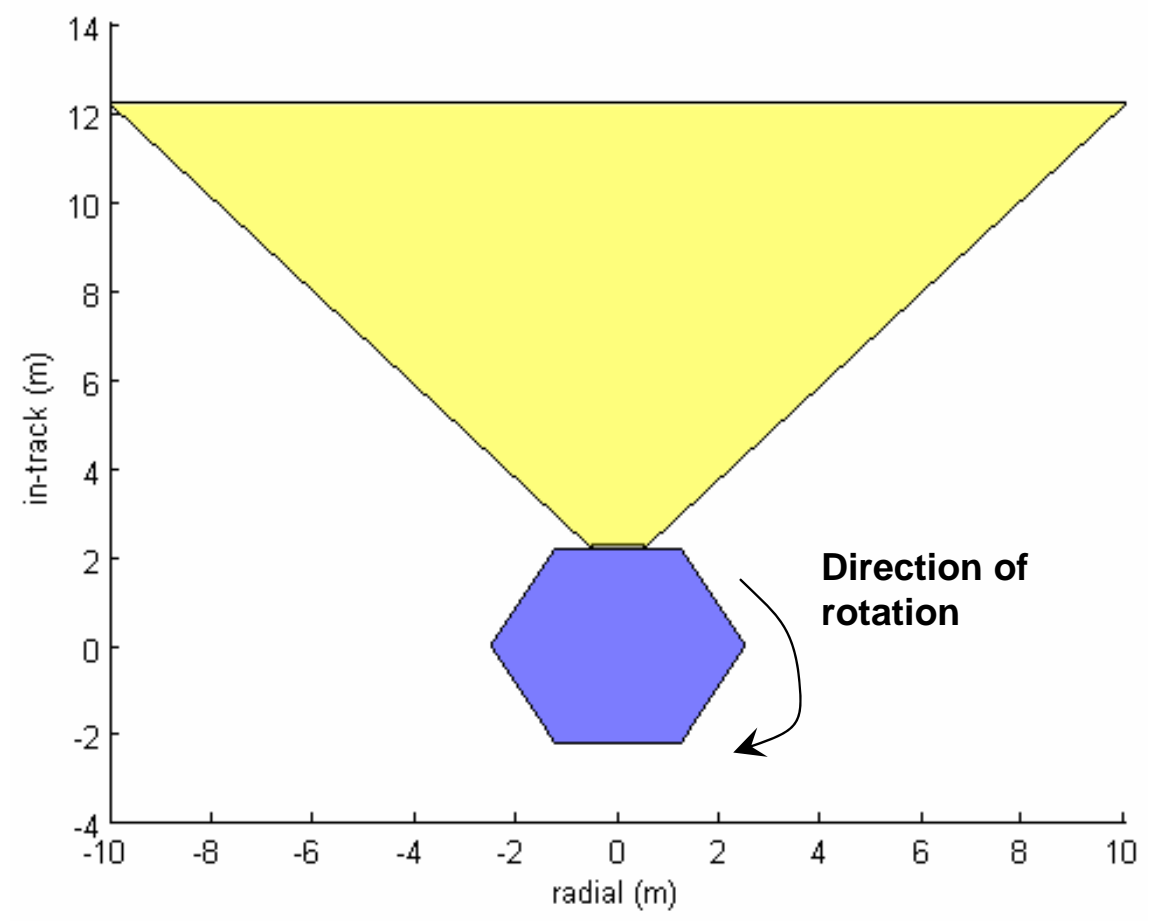

Fig. 2: Radial/in-track view of rotating target spacecraft and docking configuration 
situation for trajectories generated with safety constraints. In this case, none of the failure trajectories impact the target spacecraft. An apparent violation of the constraint boundary is visible in the lower left-hand corner of the figure. This type of corner violation is possible in the MILP framework, because only the discrete points are constrained, not the trajectory between discrete points. Reference 28 gives an approximation for the amount a constraint should be enlarged to ensure that any violations will not intersect the constrained region. As in the case without safety, several of the failure trajectories overlap. The fuel costs (measured in $\Delta V$ ) of the trajectory with no safety guarantees and the trajectory with safety are $1.29 \mathrm{~mm} / \mathrm{s}$ and $1.41 \mathrm{~mm} / \mathrm{s}$, respectively. Hence, in this case, imposing safety requires results in just under a $10 \%$ increase in fuel use. To put these numbers in context, an optimized approach constrained to follow a V-bar trajectory (strictly in-track) would use $37.7 \mathrm{~mm} / \mathrm{s}$ of fuel.

An approximate method for bounding the optimized numbers would be to consider an approach based on introducing in-track drift and arriving at the docking port after a full planning horizon, with no other constraints. In this case, the planning horizon is a full orbit, with no initial radial offset and no initial velocity, an initial thrust in the in-track direction will introduce a secular drift into the relative orbit. Over the course of an orbit this secular cause the in-track position to shift by

$$
\Delta y=\Delta v_{y} 6 \pi / n
$$

For the example in this section, the amount of fuel required for this maneuver would be $0.93 \mathrm{~mm} / \mathrm{s}$. An approximate upper bound on the fuel number could be obtained by forming a constrained problem similar to the LP, but forced to follow a strict in-track (V-bar) trajectory. If the 2-norm of fuel use is minimized instead of the correct 1-norm metric and the thruster inputs are not constrained, this problem can be solved using a pseudo-inverse. By constraining the radial position state at each step $k$ in the planning horizon to be zero and the final in-track position to lie on the edge of the docking port, the following equality constraints are formed

$$
A_{Q}\left[\begin{array}{c}
\mathbf{u}_{0} \\
\vdots \\
\mathbf{u}_{k-1}
\end{array}\right]=b_{Q} \quad \text { with } A_{Q}=\left[\begin{array}{ccccc}
H_{x} \Gamma_{1} & 0 & \ldots & 0 & 0 \\
H_{x} \Gamma_{2} & 0 & \ldots & 0 \\
H_{x} \Gamma_{N-1} & 0 \\
H_{x} \Gamma_{N} \\
H_{y} \Gamma_{N}
\end{array}\right], \quad b_{Q}=\left[\begin{array}{c}
-H_{x} A_{d}^{0} \mathbf{x}_{0} \\
-H_{x} A_{d}^{1} \mathbf{x}_{0} \\
\vdots \\
-H_{x} A_{d}^{N} \mathbf{x}_{0} \\
-H_{y} A_{d}^{N} \mathbf{x}_{0}+y_{\text {des }}
\end{array}\right]
$$

where $H_{x}$ is a row vector that extracts the scalar radial component, $H_{y}$ is a row vector that extracts the scalar in-track component, and $y_{\text {des }}$ is the desired in-track component at step $N$. This form has $N+1$ constraints and $3 N$ inputs variables to choose. The trajectory that minimizes the 2 -norm of 


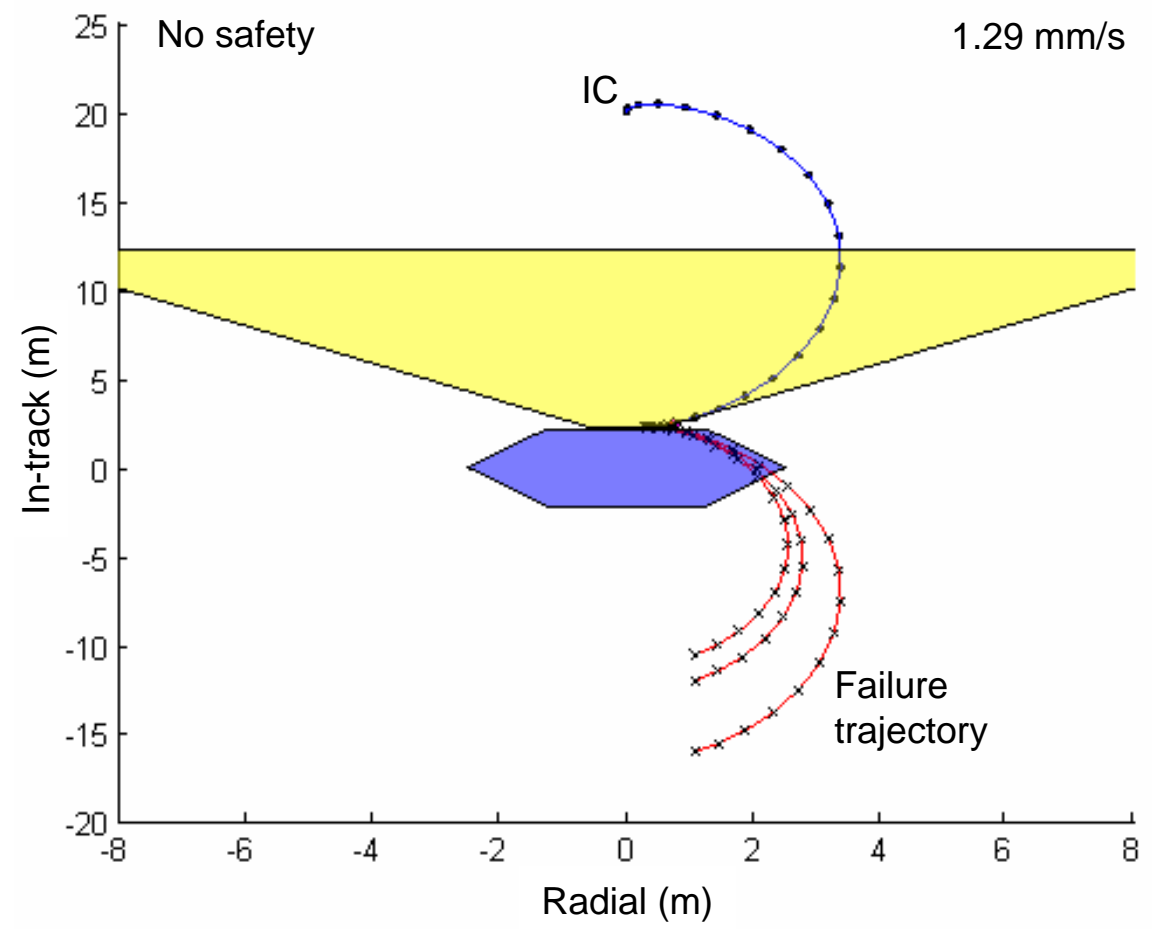

Fig. 3: Nominal trajectory planning with no safety: constraint violations occur for trajectory failures. The nominal trajectory is marked with $\bullet$ and the failure trajectories with $\times$. The failure trajectories all result in collisions with the target spacecraft.

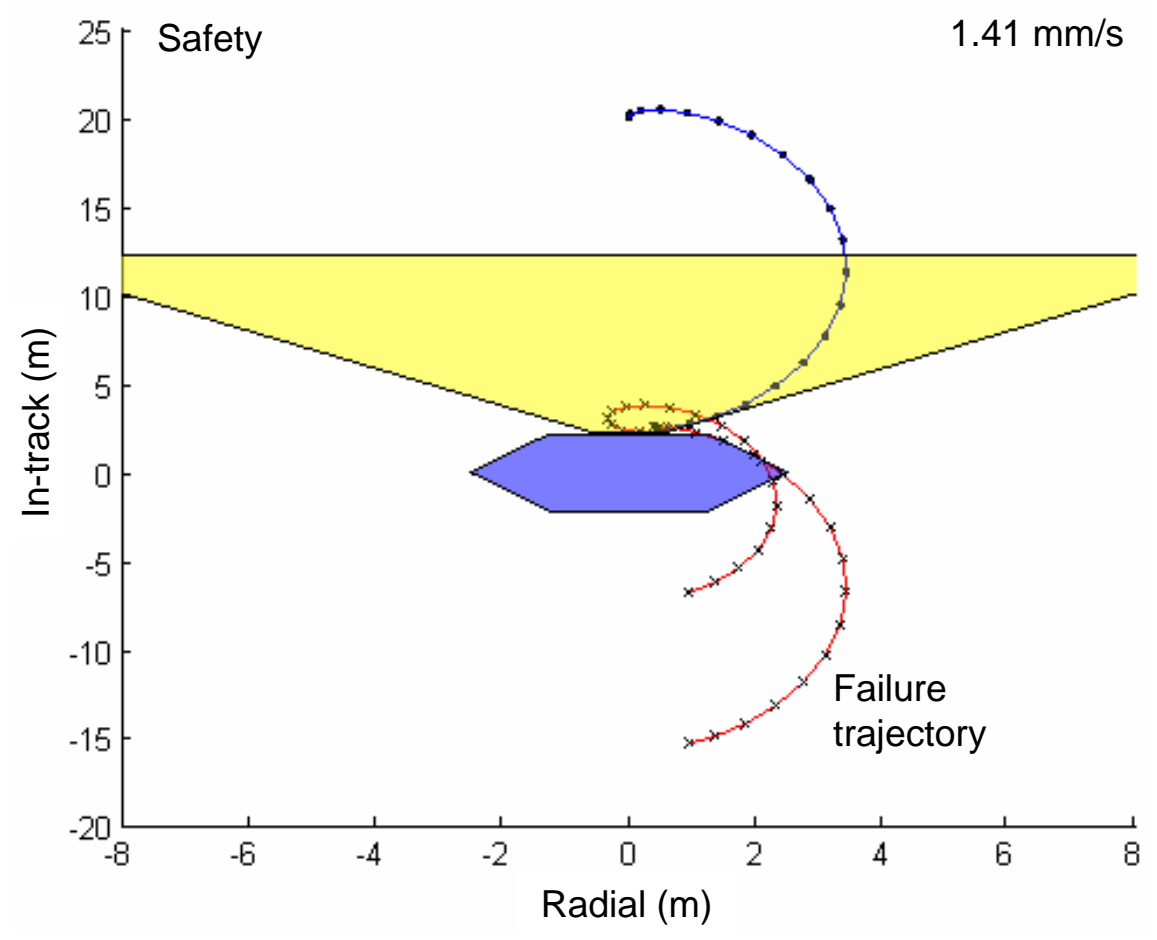

Fig. 4: Trajectory planning with safety: failed trajectories deviate around the target spacecraft, preventing collision. The nominal trajectory is marked with $\bullet$ and the failure trajectories with $\times$. 
the input vectors and meets those simple constraints is given by the pseudo-inverse solution of ${ }^{29}$

$$
\left[\begin{array}{cc}
I_{3 N, 3 N} & A_{Q}^{\prime} \\
A_{Q} & 0_{N+1}
\end{array}\right]\left[\begin{array}{c}
\mathbf{u}_{0} \\
\vdots \\
\mathbf{u}_{k-1} \\
\mathbf{z}
\end{array}\right]=\left[\begin{array}{c}
0_{3 N, 1} \\
b_{Q}
\end{array}\right]
$$

where $\mathbf{z}$ is a vector of $N+1$ Lagrange multipliers, $I_{p, r}$ is a $p \times r$ identity matrix, and $0_{p, 1}$ is a $p \times 1$ vector of zeros. The fuel cost of the trajectory found using this method is $39.1 \mathrm{~mm} / \mathrm{s}$, which is very close to the optimized cost of following a strict V-bar trajectory.

\section{B. Case 2: Docking Port Perpendicular to Spin Axis}

The rotating docking port case uses an identical formulation to the stationary case, however, the constraint regions are time-varying. Whereas in the stationary case, $A_{1}=A_{2}=A_{k} \forall k=1 \ldots N$, to formulate the rotating problem, the $A_{k}$ and $b_{k}$ matrices must be formed for each step of the planning horizon based on the rotation rate and, for more general cases, the motion of the target, the docking port, and the line-of-sight cone. A conceptually simple way to generate these constraints is to represent each side of an avoidance region as a plane which can be specified by three positions. These positions will remain in a plane through any rotation and thus the rotated constraint side can by found by rotating the points using rotation matrices. ${ }^{25}$ The equations of the new plane can be formed using the rotated points and then used as an inequality constraint. It is assumed that any translation and rotation motion of the target spacecraft is well-known before docking proceeds and can therefore be used to create the constraints. Future work will address the effects of uncertainty in target motion. Since all trajectory calculation and constraint forming occurs before the rendezvous maneuver begins, the calculation of trajectories and constraints, although fast for cases examined in this paper, can be computed to high accuracy using numerical integration.

For the rotation case examined in this section, the optimized trajectory no longer matches a twoimpulse V-bar approach, but is instead forced to thrust regularly to stay within the rotating LOS cone. Figure 5 shows this optimized trajectory with no safety constraints. As in the stationary case, in the absence of safety constraints, the nominal trajectory collides with the target in the event of guidance shutdowns. An alternate form with safety constraints prevents collisions for failure cases (Figure 6). In this case, even the safe trajectory appears to pass through the target, however in actuality avoids collision because of the rotational motion of the target. The fuel costs of the without safety constraints and with safety constraints are $36.2 \mathrm{~mm} / \mathrm{s}$ and $38.9 \mathrm{~mm} / \mathrm{s}$, respectively. As in the non-rotating case, the increase in fuel due to safety is minimal and the advantage is guaranteed collision avoidance for passive abort in the last quarter of the nominal path. Another example (safe trajectory in Figure 7) using a target rotating at $3 / 2$ orbital rate required 51.37 $\mathrm{mm} / \mathrm{s}$ of fuel with no safety constraint and $51.42 \mathrm{~mm} / \mathrm{s}$ of fuel with a safety constraint. 


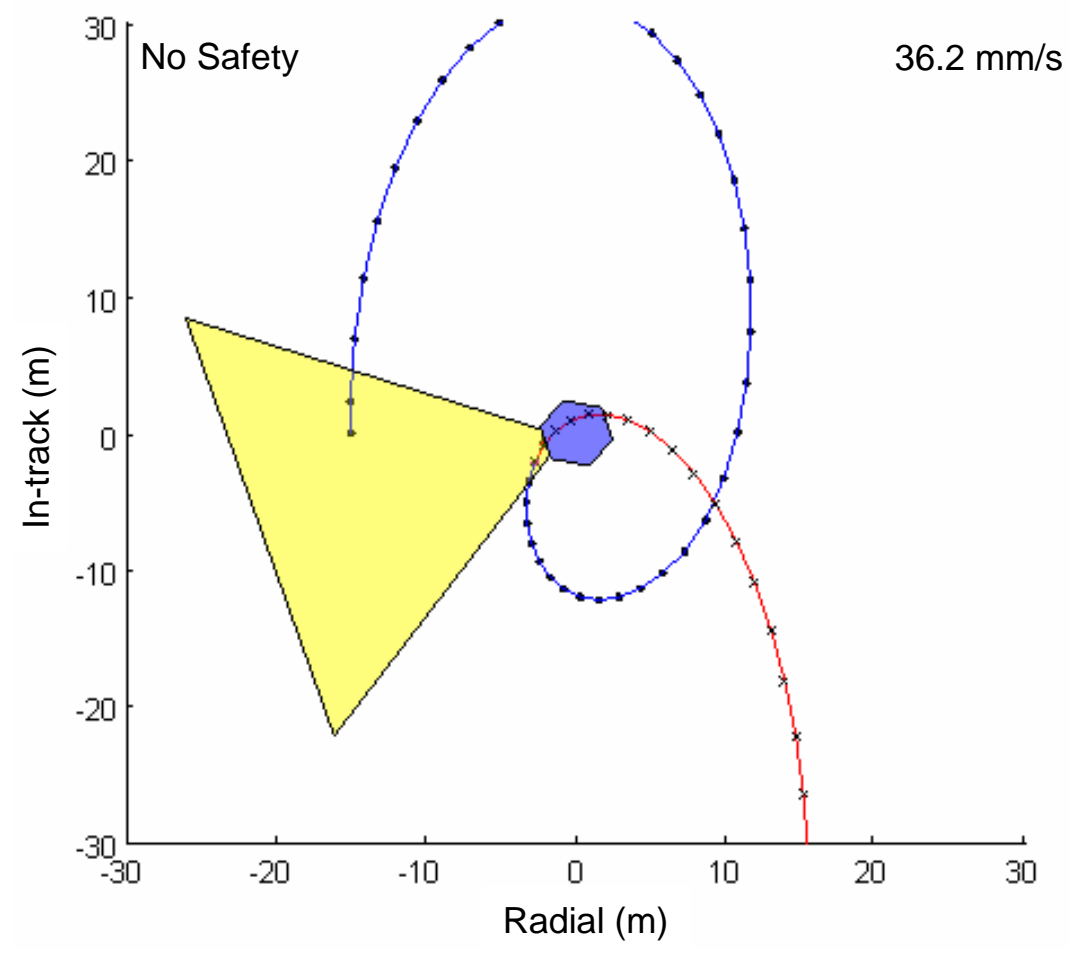

Fig. 5: Nominal trajectory planning with no safety in the rotating case: constraint violations occur for trajectory failures.

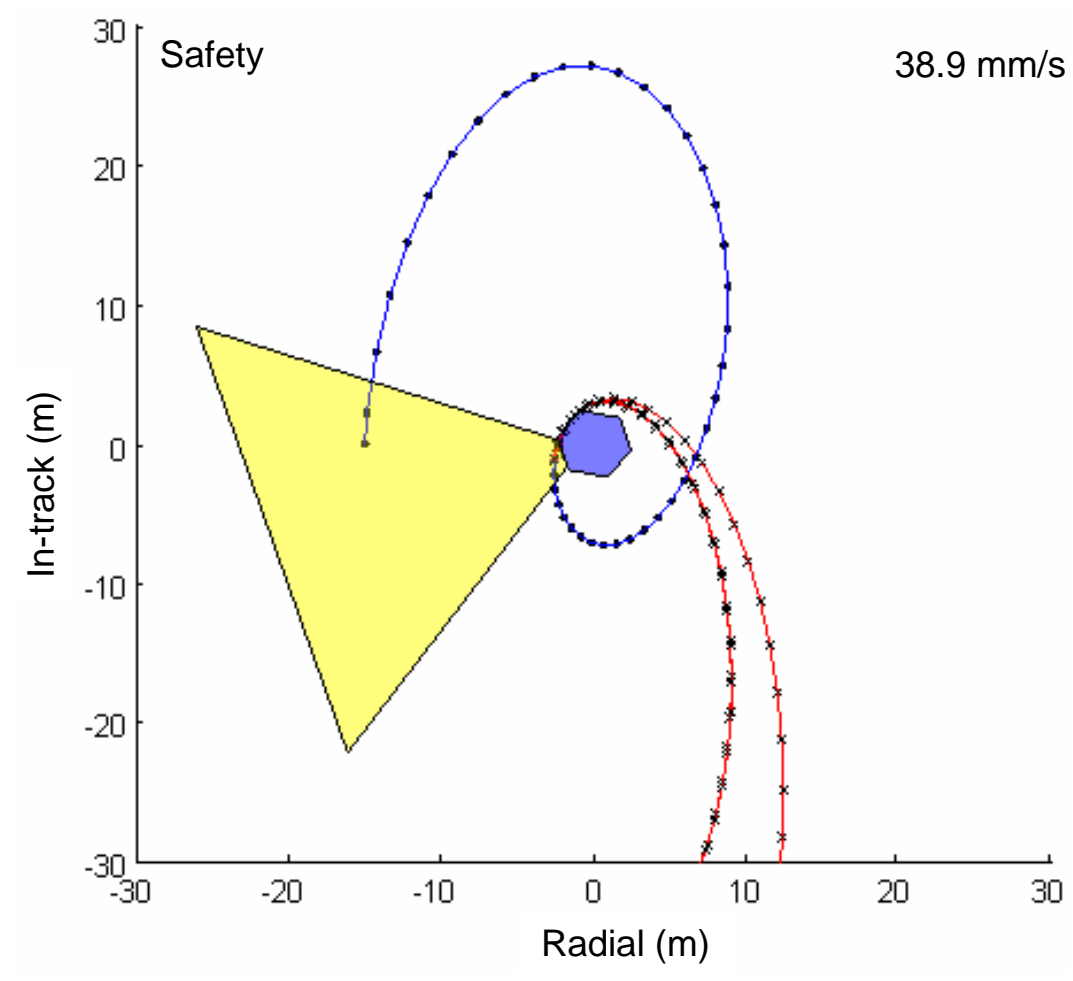

Fig. 6: Trajectory planning with safety in the rotating case: failed trajectories deviate around the target spacecraft, preventing collision 

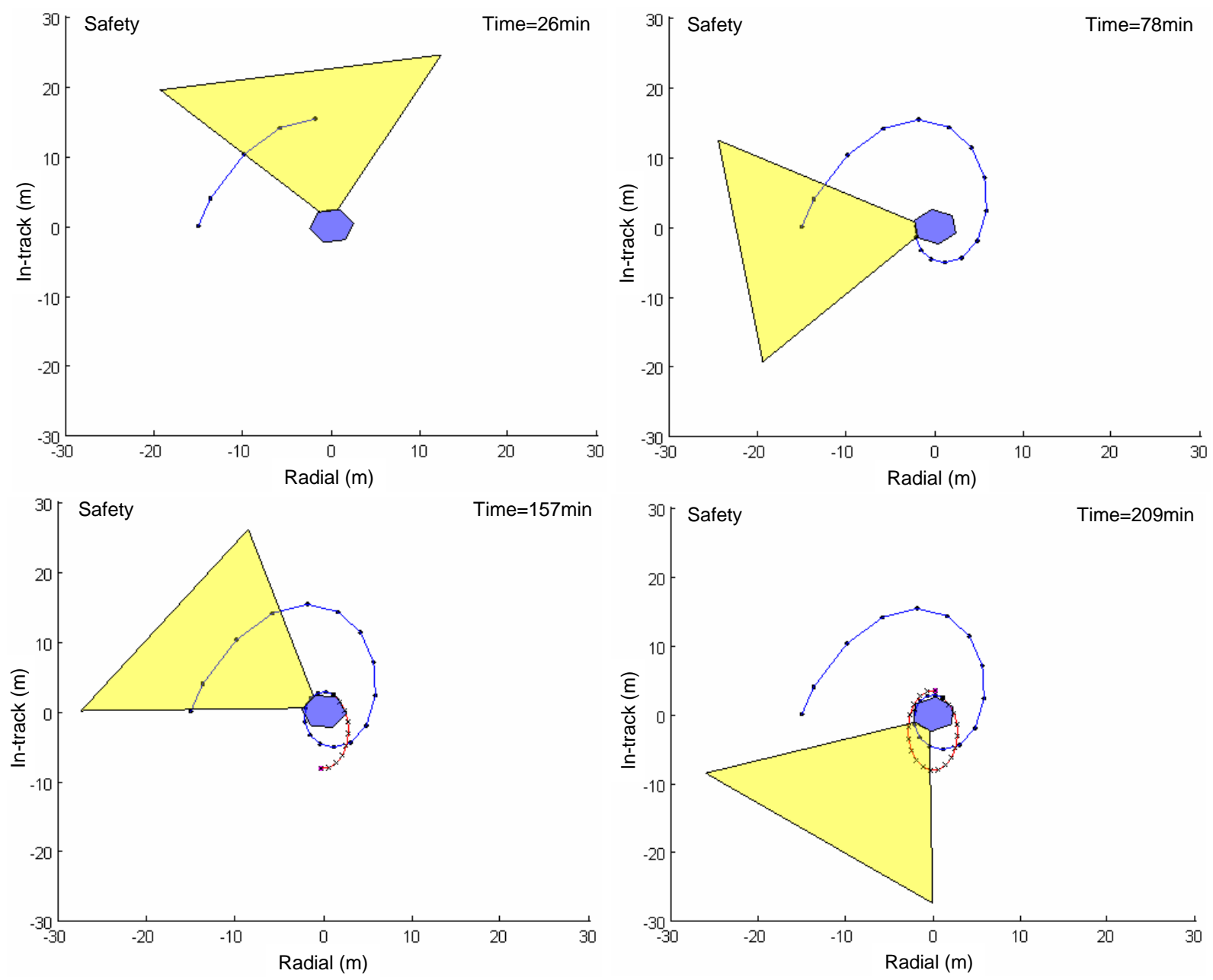

Fig. 7: Trajectory planning with safety in the fast rotating (3/2 orbital speed) case. The maneuver $\Delta V$ cost with safety is $51.42 \mathrm{~mm} / \mathrm{s}$.

\section{Invariant Formulation}

The safety formulation introduced in Section III only guarantees passive collision avoidance until the end of the safety horizon. In previous examples, the safety horizon has been fixed at one orbit. Figure 8 shows a stationary-target case where a collision would occur at the end of a 1 orbit safety horizon. If the safety horizon is extended to multiple orbits, the resulting failure trajectories will tend to either drift away from target spacecraft or create invariant orbits that neither drift toward nor away the target spacecraft. Drifting away from the target orbit is preferable to collision, however it means that fuel will need to be expended to bring the chaser near the target for any future docking attempts. Furthermore, the longer controllers wait to cancel the drift, the farther apart the two spacecraft will become, thereby creating an additional timing consideration during an anomalous event. It is preferable for the chaser to fall into an invariant orbit that is near the target, but can never, under the assumptions of Keplerian dynamics, collide. The preference for 
invariant failure orbits can be captured by the following constraint

$$
\mathbf{x}_{F T_{k}}=A_{d}^{N} \mathbf{x}_{F T_{k}} \quad \text { for } k \geq T
$$

Imposing this constraint for all possible failures times in $\mathcal{F}$, all failure orbits are guaranteed to be invariant with respect to the target. Figure 9 shows the same rendezvous problem from Figure 8, but with additional invariance constraints on failures occurring in the last quarter of the rendezvous trajectory. The resulting trajectories in with invariance constraints form circular trajectories relative to the target that they traverse once per orbit with no fuel expenditure. Some of the six failure trajectories shown in Figure 9 have overlap in cases where the optimized trajectory did not require fuel inputs. The safe trajectory with invariance used $2.93 \mathrm{~mm} / \mathrm{s}$ of fuel and the safe trajectory with invariance used $6.1 \mathrm{~mm} / \mathrm{s}$ of fuel. In this case, the invariance constraints have roughly doubled fuel requirements. However, this tradeoff may be beneficial when the danger of a collision after the safety horizon or the fuel requirements of canceling drift after a failure are taken into account. For this case, the low estimated fuel numbers from Eq. 16 predict $1.4 \mathrm{~mm} / \mathrm{s}$ of fuel required and the strict V-bar approach solution from Eq. 18 predicts $61.4 \mathrm{~mm} / \mathrm{s}$ of fuel use, which is close to the optimized V-bar solution which requires $59.2 \mathrm{~mm} / \mathrm{s}$. The cost of optimized rendezvous trajectory with no safety constraints is $2.89 \mathrm{~mm} / \mathrm{s}$.

\section{Convex formulation}

The safety constraints introduced in Section III guaranteed that the chaser states in the event of a failure would not collide with the target. The collision avoidance in those constraints is accomplished using binary variables to capture the nonconvexity of the problem. The problem with binaries was formulated as a Mixed-Integer Linear Program (MILP) and posed to a commercial solver. Solving a MILP can be a computationally intensive task and the time required to solve tends to grow very quickly with the number of discrete variables in the problem. ${ }^{20}$ The trajectory shown in Figure 4 required 9.15 seconds to solve on a $3 \mathrm{GHz}$ computer. That problem had a 20 step safety horizon, an avoidance region with 8 sides, and six inputs that were safe in the event of guidance shutdown. Each avoidance region side requires a binary variable at each step of the safety horizon and those constraints are included 6 times, each propagating forward from a different failed thruster step. Thus, implementing collision avoidance over the safety horizon for that simple example required 960 binary variables. Solving the same trajectory for a two orbit safety horizon required 73.4 seconds. Using the same horizon duration with a finer discretization step would further increase the required computation time. It is likely that online implementations would need to solve with limited computer resources and that a nonconvex implementation may be impractical for implementations requiring short discretization steps.

An alternative to the nonconvex formulation is to use a more restrictive form of collision avoidance that is convex. Instead of requiring the chaser to remain outside an avoidance region, the failure trajectories are instead constrained to remain inside a region that is known to not contain the target. This is similar to the type of convex passively safe trajectory examined for rotating 


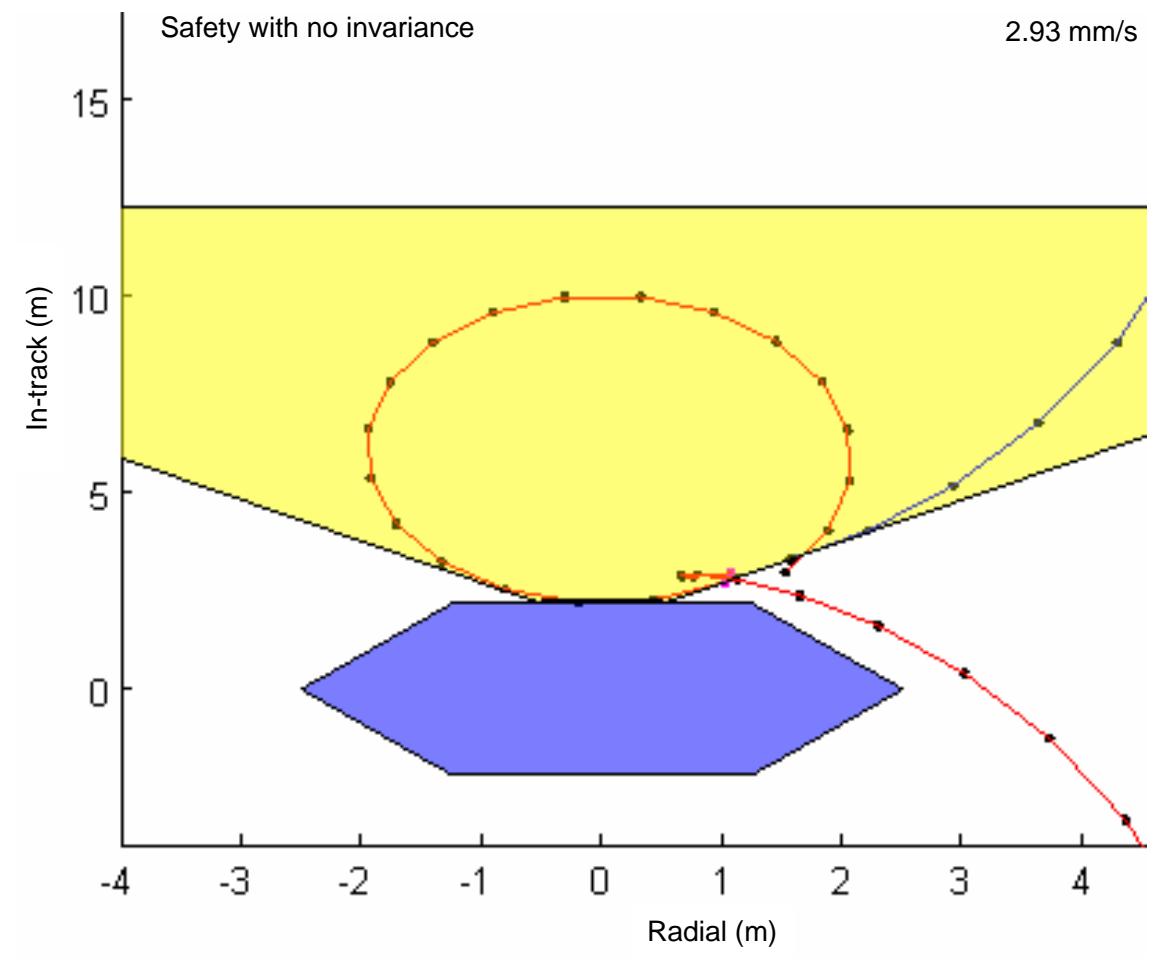

Fig. 8: Case where end of safety horizon is followed by a collision.

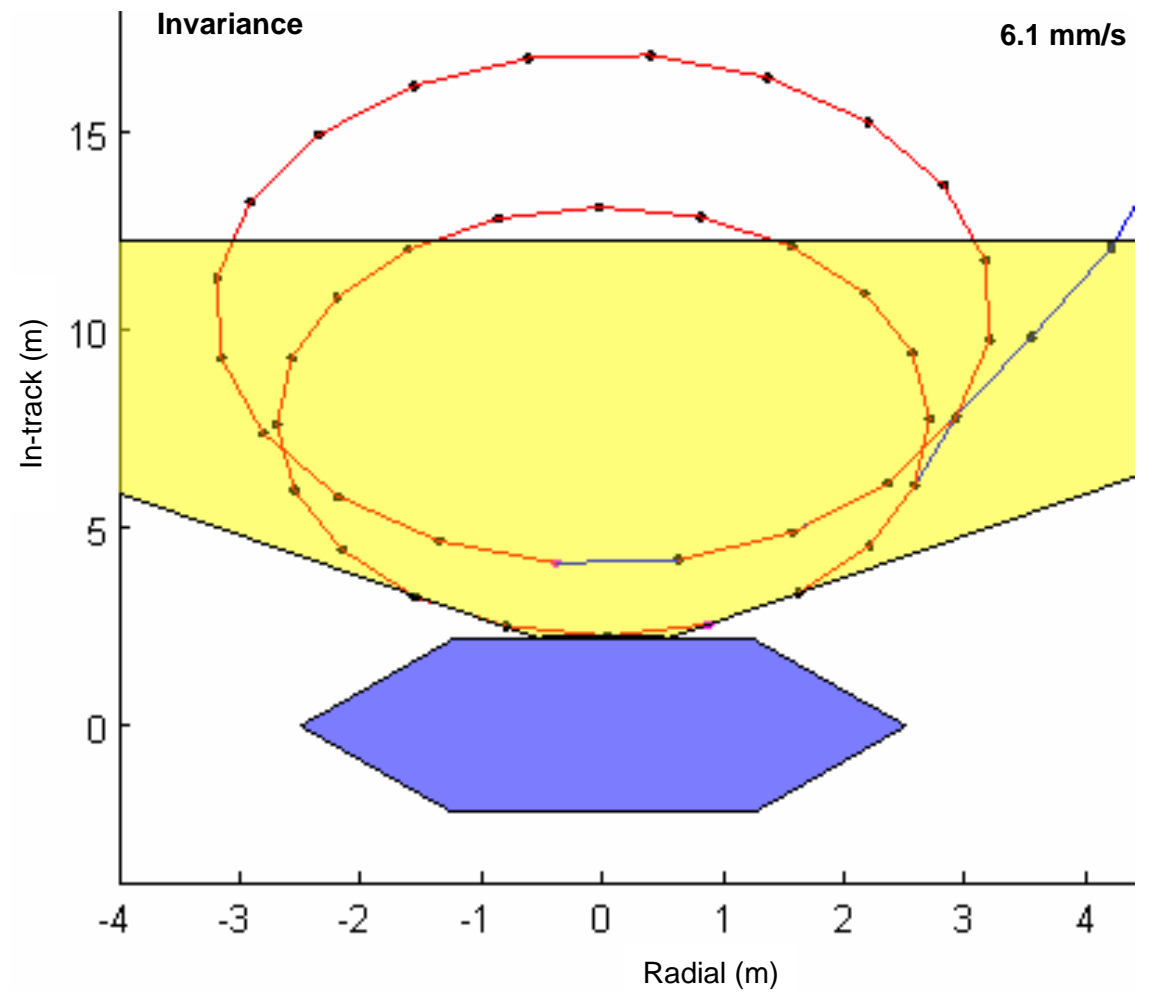

Fig. 9: Use of invariance constraints guarantees infinite horizon passive collision avoidance and prevents failure trajectories from drifting away from the target. 


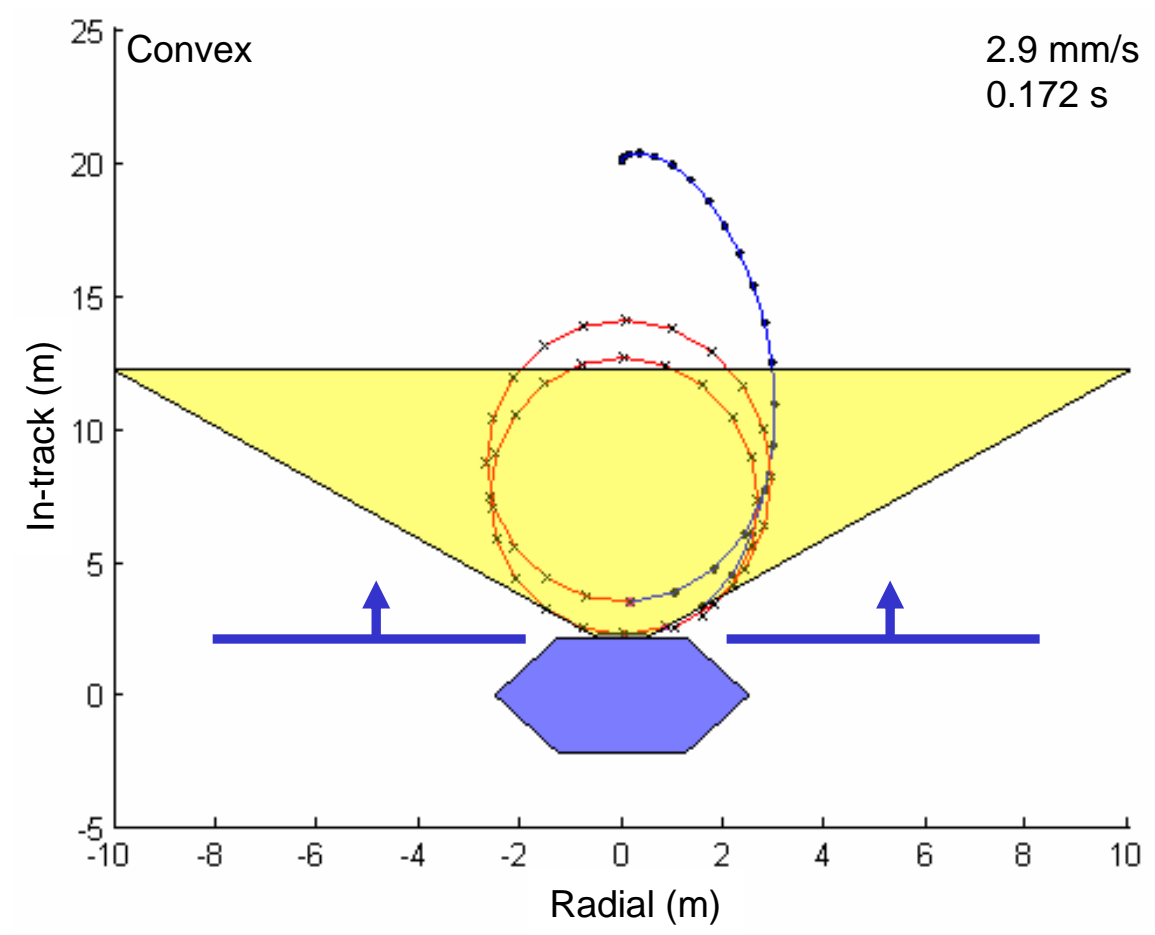

Fig. 10: Collision avoidance for failure trajectories using convex constraints indicated by arrows.

satellite capture in Ref. 14. Figure 10 shows an example of the optimized trajectory for the rendezvous problem in Figure 4, but instead solved as a linear program (LP) using the convex safety constraints

$$
H_{y} \mathbf{x}_{F T_{k}} \geq y_{\min } \forall k \in\{T+1 \ldots N+S\}
$$

where $y_{\min }$ is the maximum in-track position of the target spacecraft. The nonconvex case results in failure trajectories that are not permitted in the convex case and as a result, there is a fuel penalty for imposing convexity. The nonconvex cases requires $1.41 \mathrm{~mm} / \mathrm{s}$ of $\Delta V$ and the convex case requires $2.9 \mathrm{~mm} / \mathrm{s}$. The more restrictive area in which failure trajectories can lie caused the required fuel to increase by a factor of 2.06 . However, the time required to compute the convex trajectory was only 0.172 seconds, a decrease from the nonconvex case by a factor of 53 . In cases where it is impractical to dedicate significant computational resources to planning, it may be desirable to trade the fuel optimality of the more general MILP formulation for the speed of the LP formulation.

\section{Conclusion}

Safety in autonomous spacecraft rendezvous trajectory design allows passive abort with guaranteed collision avoidance for a class of anomalous system behavior. This paper examined several online optimization formulations that guarantee safety and determined that the additional fuel costs are comparatively small, particularly relative to commonly considered suboptimal trajectories. Additional restrictions to guarantee failure trajectories that minimize drift and guarantee infinite horizon passive collision avoidance were introduced shown to require fuel use on the same 
order of magnitude as the unsafe optimized solutions. Approximate analytic methods for creating upper and lower bounds on the expected fuel use of several examples yielded accurate estimates compared to optimized fuel costs. A convex formulation of the safety problem was introduced and demonstrated using twice as much fuel, but more than 50 times less computation time than the nonconvex formulation.

\section{Acknowledgments}

This work was funded under Cooperative Agreement NCC5-729 through the NASA GSFC Formation Flying NASA Research Announcement. Any opinions, findings, and conclusions or recommendations expressed in this material are those of the author(s) and do not necessarily reflect the views of the National Aeronautics and Space Administration.

\section{References}

${ }^{1}$ D. J. Zimpfer, P. S. Spehar, F. Clark, C. D' Souza, and M. Jackson, "Autonomous rendezvous and capture guidance, navigation and control," Flight Mechanics Symposium, (Goddard Space Flight Center, Greenbelt, Maryland), Session 3, Paper 7, October 18-20, 2005.

${ }^{2}$ M. E. Polites, "Technology of Automated Rendezvous and Capture in Space," AIAA Journal of Spacecraft and Rockets, vol. 36, no. 2, March-April 1999, p. 280-291.

${ }^{3}$ I. Kawano, M. Mokuno, T. Kasai, T. Suzuki, "Result and evaluation of autonomous rendezvous docking experiments of ETS-VII," Proceedings of the AIAA Guidance, Navigation and Control Conference, Aug 1999. AIAA1999-4073.

${ }^{4}$ T. E. Rumford, "Demonstration of Autonomous Rendezvous Technology (DART) Project Summary," Proceedings of SPIE, Volume 5088, pp.10-19, 2003.

${ }^{5} \mathrm{~K}$. Young, "Autonomous rendezvous in space becomes hit and run," New Scientist Space, online at http://www.newscientistspace.com/article/dn7303, last accessed Jan. 2006.

${ }^{6}$ B. Berger, "Fender Bender: NASA's DART Spacecraft Bumped Into Target Satellite," Space.com, online at http://www.space.com/missionlaunches/050422_dart_update.html, last accessed Jan. 2006.

7 "Overview of the DART Mishap Investigation Results," NASA.gov, online at http://www.nasa.gov/mission_pages/dart/main/index.html, last accessed May 2006.

${ }^{8}$ A. G. Richards, T. Schouwenaars, J. P. How, E. Feron, "Spacecraft Trajectory Planning With Collision and Plume Avoidance Using Mixed Integer Linear Programming," AIAA Journal of Guidance, Control and Dynamics, vol. 25, pp.755-764, Aug 2002.

${ }^{9}$ I. Garcia and J. P. How, "Trajectory Optimization for Satellite Reconfiguration Maneuvers with Position and Attitude Constraints" Proceedings of the IEEE American Control Conference, June 2005, pp.889-895

${ }^{10}$ P. K. C. Wang, M. Mokuno, and F. Y. Hadaegh, "Formation Flying of Multiple Spacecraft with Automatic Rendezvous and Docking Capability," AIAA Guidance, Navigation, and Control Confrence, Austin, TX, Aug 11-14, 2003.

${ }^{11}$ W. Fehse. Automated Rendezvous and Docking of Spacecraft. Cambridge University Press, 2003.

${ }^{12}$ B. Naasz, "Safety Ellipse Motion with Coarse Sun Angle Optimization," Flight Mechanics Symposium, (Goddard Space Flight Center, Greenbelt, Maryland), 2005.

${ }^{13}$ S. Jacobsen, C. Lee, C. Zhu, S. Dubowsky "Planning of safe kinematic trajectories for free flying robots approaching an uncontrolled spinning satellite," DETC2002/MECH, Montreal, Canada, 2002.

${ }^{14}$ S. Matsumoto, S. Dubowsky, S. Jacobsen, Y. Ohkami, "Fly-by Approach and Guidance for Uncontrolled Rotating Satellite Capture," AIAA Guidance, Navigation, and Control Confrence, Austin, TX, Aug 11-14, 2003. 
${ }^{15}$ T. Schouwenaars, J. P. How, and E. Feron, "Decentralized Cooperative Trajectory Planning of Multiple Aircraft with Hard Safety Guarantees," Proceedings of the AIAA Guidance, Navigation and Control Conference, Aug 2004. AIAA-2004-5141.

${ }^{16}$ C. Tomlin, I. Mitchell, R. Ghosh, "Safety Verification of Conflict Resolution Maneuvers," IEEE Transactions on Intelligent Transportation Systems, vol 2., no 2., June 2001, p.110.

${ }^{17}$ M. Tillerson and J. P. How, "Analysis of the Impact of Sensor Noise on Formation Flying Control," Proceedings of the 2001 American Control Conference, Arlington, VA, June 25-27, 2001.

${ }^{18}$ L.S. Breger and J.P. How, "J $J_{2}$-Modified GVE-Based MPC for Formation Flying Space," AIAA GNC Conf., August 2005.

${ }^{19}$ G. Franklin, J. Powell, and M. Workman, "Digital Control of Dynamic Systems," Third Edition,AddisonWesley, 1998.

${ }^{20}$ D. Bertsimas and J. N. Tsitsiklas, Introduction to Linear Optimization, Athena Scientific, Belmont, 1997.

${ }^{21}$ G. W. Hill, "Researches in Lunar Theory," American Journal of Mathematics, Vol. 1, 1878, pp. 5-26,129$147,24-260$.

${ }^{22}$ M. Tillerson, G. Inalhan, and J. P. How, "Co-ordination and control of distributed spacecraft systems using convex optimization techniques," International Journal of Robust and Nonlinear Control, Vol.12, John Wiley \& Sons, 2002, p. 207-242.

${ }^{23}$ A. G. Richards, "Robust Constrained Model Predictive Control," PhD Thesis, Massachusetts Institute of Technology, November 2004.

${ }^{24}$ Alexander B. Roger and Colin R. McInnes, "Safety constrained freeflyer path planning at the international space station," Journal of Guidance, Control, and Dynamics, 23(6):971979, NovemberDecember 2000.

${ }^{25}$ D. T. Greenwood, Advanced Dynamics, Cambridge University Press, Cambridge, 2003.

${ }^{26}$ M. Kaplan. Modern Spacecraft Dynamics and Control. Wiley, 1976.

${ }^{27}$ J. P. How, R. Twiggs, D. Weidow, K. Hartman, F. Bauer, "Orion - A low-cost demonstration of formation flying in space using GPS," Proceedings of AIAA/AAS Astrodynamics Specialist Conference and Exhibit, Boston, MA, Aug. 10-12, 1998, Collection of Technical Papers (A98-37348 10-13), Reston, VA, American Institute of Aeronautics and Astronautics, 1998, p. 276-286.

${ }^{28}$ Y. Kuwata, Real-time Trajectory Design for Unmanned Aerial Vehicles using Receding Horizon Control, S.M. Thesis, Dept. of Aeronautics and Astronautics, MIT, Jun. 2003.

${ }^{29}$ G. Strang. Introduction to Applied Mathematics. Wellesley-Cambridge Press, 1986. 